\title{
Santiago 1872-1875: UN PLANO PARA EL PLAN DE VICUÑA MACKENNA ${ }^{1}{ }_{\text {(FIG. I) }}$
}

\author{
Germán Hidalgo, Wren Strabucchi, José Rosas, Magdalena Montalbán, Germán Guzmán \\ Pontificia Universidad Católica de Chile
}

\begin{abstract}
A casi 150 años de su implementación, aún se sabe relativamente poco de los alcances de la transformación de Santiago emprendida por Benjamín Vicuña Mackenna entre 1872 y 1875. En ello han colaborado tanto la escasa documentación gráfica que ha llegado hasta nosotros como la generalidad de las investigaciones realizadas ahora, pues la mayoría de ellas ha dado por descontado que el plano de Santiago de Ernesto Ansart de 1875 representa ese plan de transformación. Respondiendo a lo anterior, se ha elaborado una cartografía que analiza la ordenación morfotipológica y el trazado urbano de esa ciudad con el fin de dilucidar dos importantes cuestiones: ¿cuál era la realidad urbanística que registraba Santiago hacia 1872? y ¿cuáles fueron los principios, condiciones y elementos que orientaron el plan de transformación? Aventuramos que la transformación de Santiago de 1872 fue guiada por una especial actitud del intendente: la de situarse entre la concreta realidad de la ciudad y las obras necesarias para su transformación, pero sin el plano que registrará lo existente y sobre el cual proyectar la ciudad futura. Por esta razón, se debe dejar de lado el plano de Ansart, pues, contrariamente a lo que se piensa, es más lo que oculta que lo que revela de esa ciudad en transformación. Precisar esa realidad y conocer ese plan es el objetivo de la cartografía propuesta y de este artículo que la acompaña.
\end{abstract}

Palabras clave: Ansart, cartografía urbana, transformación de Santiago, Vicuña Mackenna.

En la historiografía de la urbanización de nuestras ciudades, la representación cartográfica es aún una tarea pendiente. Y aunque ha habido importantes contribuciones sobre la evolución de las ciudades latinoamericanas de parte de diversos autores (Hardoy, I978; Cicutti, 2012; Fabeluke, 202I), el abordaje de mapas y planos, como portadores de lecciones sobre la ciudad, no ha sido relevante en los estudios de historia urbana, más allá de su uso incidental, complementario o auxiliar. Ello se debe, probablemente, al laborioso trabajo que exige la integración del recurso cartográfico en la visualización y conceptualización de la organización espacial y, en consecuencia, a las dificultades que conlleva la representación de la ciudad y el territorio en una escala que permita el examen atento de las transformaciones urbanas sin merma en la visualización del conjunto y en la comprensión de los procesos urbanos.

Creemos que en una investigación que trata sobre cómo representar eficazmente la forma general de la ciudad, uno de los desafíos más importantes es avanzar en la comprensión tanto de la historia de la forma de dicha ciudad como de la complejidad de su desarrollo y persistencia en el tiempo. Ello requiere entender las relaciones entre las partes constituyentes de la ciudad, vale decir, el parcelario, los espacios públicos y las edificaciones, como también aquellos nuevos conceptos y operaciones que se proponen para transformarla.

En esta línea, en la transformación de Santiago de Vicuña Mackenna de I872 se debe distinguir entre la representación cartográfica necesaria para explicar el estado de la estructura urbana y el instrumento necesario para ejecutar los cambios.

Dada la estrecha relación de trabajo y colaboración entre Vicuña Mackenna y Ernesto Ansart, se han dado por descontadas muchas concordancias entre el modelo de ciudad que tenía en mente el intendente para el plan de transformación y el plano que finalmente publicó el ingeniero en $1875^{2}$. Ello se debe a que pocas veces en la historia urbana de Santiago se ha puesto de relieve la necesidad de simultanear plano y plan a la hora de intervenir radicalmente en la ciudad.

Pero en este caso fue diferente. Mientras Vicuña Mackenna realizaba su propuesta en base a las dinámicas urbanas existentes en I872, y la consecuente necesidad de definir una nueva planta para la ciudad, Ernesto Ansart representó las calles y manzanas de modo esquemático y homogéneo, como si en toda su extensión alcanzara el mismo grado de consolidación. Es decir, si el primero trabajaba sobre la ciudad real, con sus tensiones y conflictos, esbozando una propuesta de nuevos límites, de cambio de escala y de forma, el segundo iba a concretar un plano de carácter informativo, para desenvolverse en ella, haciendo énfasis en su organización administrativa, señalando las subdelegaciones, los cuarteles de incendio, y especificando la ubicación de buzones de correo, hoteles y escuelas (Hidalgo, Strabucchi, Montalbán, 2020). Es decir, nos enfrentamos a dos visiones divergentes, pero que sin embargo intentaban medirse con una misma realidad urbana en transformación y, por tanto, dispersa, heterogénea y fragmentaria. En este artículo intentamos, pues, evidenciar el nivel de evolución histórica registrado por la ciudad en un período muy concreto de su desarrollo (I872), junto a la concepción de una ciudad nueva, soportada en las operaciones y las obras necesarias para hacerla posible (I872-1875). Tal es el objetivo de este trabajo, que se decanta, sintéticamente, en una única construcción cartográfica que describe el estado de la ciudad en ese período y el plan concebido para su transformación.

\section{La cartografía urbana como objeto y método} de investigación

La transformación de Santiago de I872 ha sido estudiada ininterrumpidamente desde hace ya varias décadas, pero
FIGURA 1. (portada y contraportada) Detalle del plano de Santiago 1872-1875, escala 1: 5.000, sector cerro Santa Lucía. Fuente: elaboración propia. Fondecyt $N^{\circ} 1191393$
I. Este artículo es un producto de la investigación Fondecyt N I191393, "Santiago I875: Desmontando el plano de Ernesto Ansart y el plan de transformación de Benjamín Vicuña Mackenna: entre la modernización de la ciudad capital y el emergente urbanismo residencial" (2019-2022).

2. Ernesto Ansart, Plano de Santiago. Con las divisiones políticas $i$ administrativas, los ferrocarriles Urbanos $i$ a Vapor, Establecimientos de instrucción de beneficencia i religiosos. Con los proyectos de canalización del río, Camino de Cintura, Ferrocarriles etc., I: 6.666 (ImpMonrocq, 1875). 

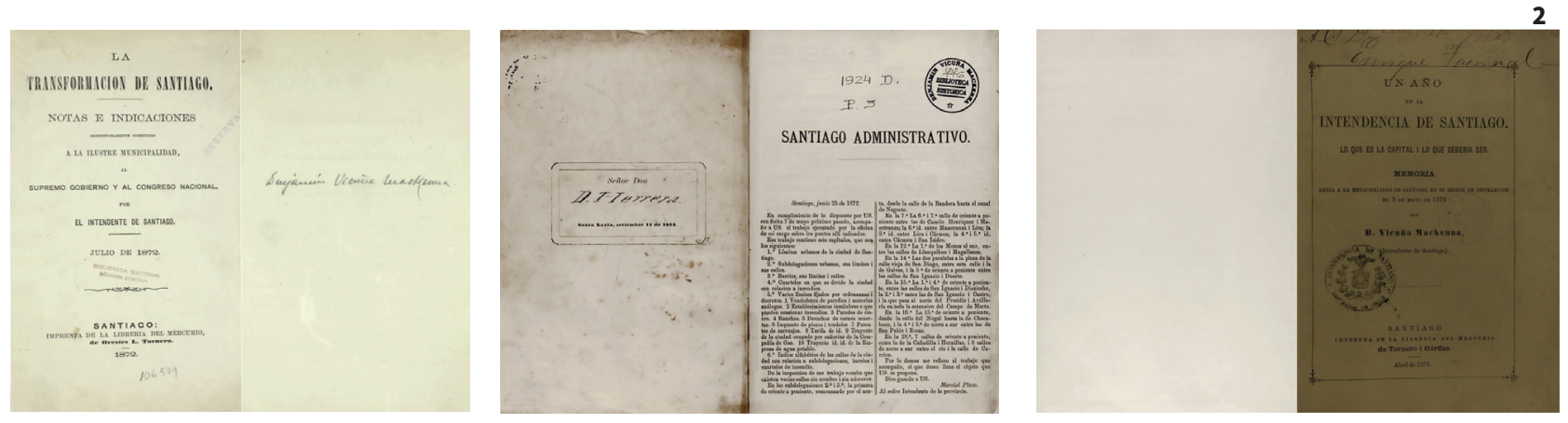

por su indiscutible trascendencia y no menor controversia todavía goza de gran actualidad. Se ha estudiado desde distintos ángulos y con diversos niveles de profundidad (Echaiz, I975; Rodriguez, I984; de Ramón, I985; Astaburuaga, I993; Rodríguez, I995; Correa, 2015; Montealegre, 20I7; Vyhmeister-Fábregas, 2019), sin embargo, hasta ahora, estos estudios no han utilizado el dibujo como método de investigación (Solá-Morales y Parcerisa, I980; Parcerisa, 2012).

Bien por el contrario, el tema se ha explicado parcialmente y sin considerar la totalidad de las fuentes, como la amplitud de textos que Vicuña Mackenna le dedica a la ciudad de Santiago, además de los diferentes informes realizados por sus colaboradores (FIG. 2). Como ya se ha mencionado, un elemento que ha incidido particularmente en esta confusión es la errónea asociación del plano de Ernesto Ansart con el plan de transformación de Vicuña Mackenna (Hidalgo, Strabucchi, Montalbán, 2020). Su aspiración de mostrar una ciudad moderna, homogénea y coherente invisibilizó a aquella que Vicuña Mackenna quiso transformar. Esquemático en general, el plano de Ansart no distingue las zonas centrales, entonces ya bien consolidadas, de aquellas otras periféricas cuyos suelos transitaban de lo rural a lo urbano (de Ramón, 1985), como tampoco muestra el antiguo y obsoleto sistema de acequias que a mal traer seguía en funcionamiento. Junto a esto, el plano de Ansart no tiene la intención de visibilizar la tensión existente entre la ciudad capital y los emergentes urbanismos residenciales de los suburbios; una distinción relevante que caracterizaba la producción de la ciudad en este período. Pero, en cambio, sí se esmeraba en representar los proyectos, aunque no todos. Como se señala en la viñeta, el plano consigna la canalización del río, el Camino de Cintura, la apertura de calles tapadas y otras nuevas, además de las vías del ferrocarril, a vapor y urbano; un conjunto de intervenciones que no siempre coinciden con el que señalan las fuentes. No hay cómo corroborar, por ejemplo, el trazado del Camino de Cintura, particularmente en las secciones norte y poniente, ya que no se ejecutaron y en ningún otro lugar se señala cuál fue la solución definitiva. Por último, se debe hacer notar que el plano de Ansart se publicó a mediados de i875 (Barros Arana, 1875), meses después de que Vicuña Mackenna concluyera su gestión, hecho que viene a confirmar la independencia de sus objetivos ${ }^{3}$.

Si fuera posible sintetizar el conjunto de las propuestas de Vicuña Mackenna en una sola gran intención, esta sería la de dar a Santiago una nueva planta urbana, cuya concepción y lógica derivaba de una serie de operaciones encadenadas para dotarla de una coherencia formal. Visto así, era un desafío enorme, sólo comparable al acto de su fundación. Pero aun consciente de la magnitud de esta tarea, Vicuña Mackenna emprendió otra igual de desafiante: elaborar el "plano definitivo" de Santiago, consistente en un levantamiento exhaustivo que permitiera

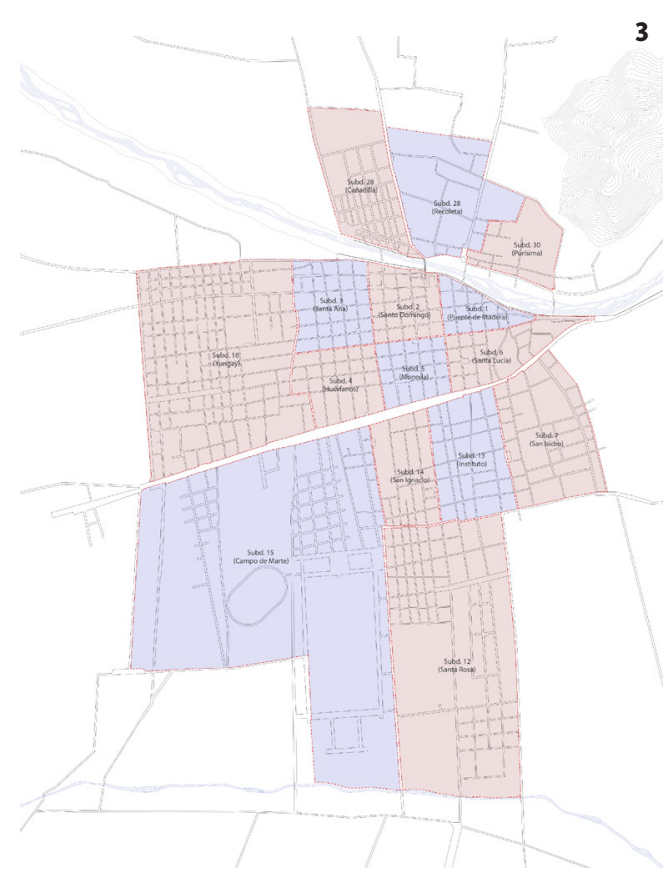

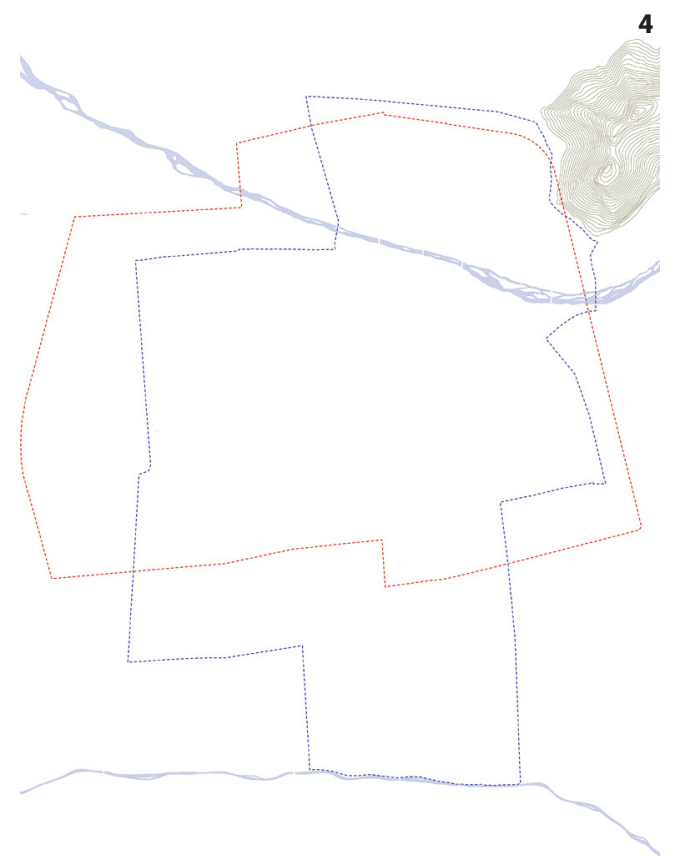

FIGURA 2

Portadas de los documentos de Benjamín Vicuña Mackenna y colaboradores. Fuente: Biblioteca Nacional de Chile y Museo Nacional Benjamín Vicuña Mackenna.

\section{FIGURA 3}

Subdelegaciones urbanas según el informe de Marcial Plaza, Santiago administrativo, de 1872 Fuente: elaboración propia. Fondecyt $\mathrm{N}^{\circ}$ 1191393.

\section{FIGURA 4}

Limite urbano de Santiago en 1872 y en 1873. En azul se traza el límite urbano Santiago en 1872 según el informe de Marcial Plaza. Al sur el límite queda perfectamente definido por el Zanjón de la Aguada y al poniente por las vías del ferrocarril y la Quinta Normal. Al norte y al oriente los límites son menos rotundos. En rojo se traza el límite urbano de Santiago según la Ley de 1873. En gran parte de la ciudad el límite quedó definido por el Camino de Cintura. Al norte y al poniente los límites quedaron sin definir, por la misma ausencia en estos sectores del camino. Fuente: elaboración propia. Fondecyt $N^{\circ} 1191393$.

\footnotetext{
3. Diego Barros Arana menciona, el $\mathrm{I}^{\mathrm{o}}$ de agosto de I875, que el plano de Ernesto Ansart había sido publicado en París recientemente.

4. La Ley de Subdelegaciones del Departamento de Santiago (26 de diciembre de I873) modificó el perímetro de la ciudad al reordenar las subdelegaciones urbanas y rurales. Así, el límite de la ciudad (dado por las subdelegaciones urbanas) coincidió con el Camino de Cintura. Consecuencia de esto fue la
} 


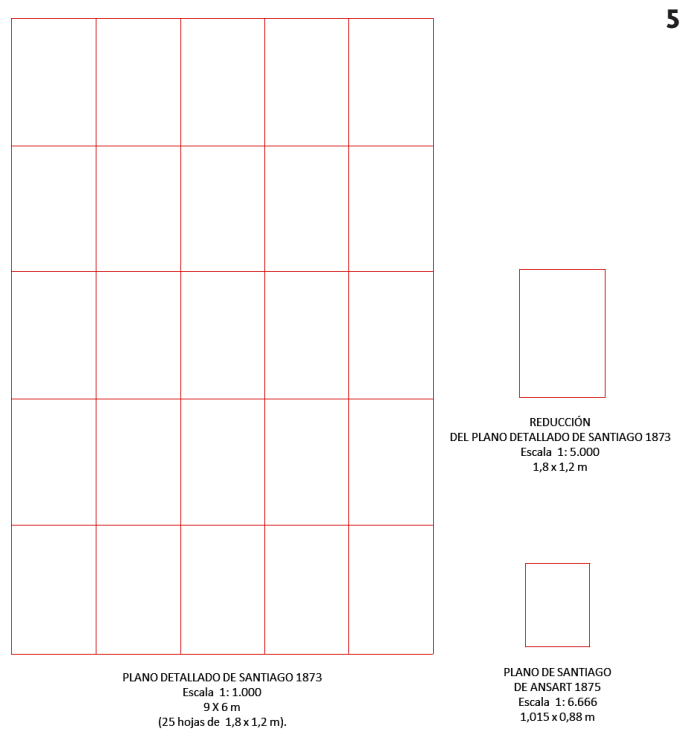

comprender la ciudad, planificar las obras de transformación e incorporar las modificaciones necesarias (Vicuña Mackenna, I873a).

Las escalas I: I.000 y i: 5.000 mencionadas por Vicuña Mackenna (I873a) y Ansart para el "plano definitivo” evidencian la originalidad de este trabajo, ya que nunca antes se había cartografiado la ciudad con este grado de detalle. Vicuña Mackenna señalaba, en distintos documentos, que este plano debía estar a la altura de ese gran desafío de transformar Santiago (Vicuña Mackenna, 1872). Con ello quedaba plenamente confirmada la necesidad de una cartografía realizada rigurosamente, como registro de la realidad existente e instrumento posibilitador de la transformación.

Lamentablemente, esta idea, que hoy podemos llamar plano-plan, no se pudo concretar, derivando así en un plan de transformación carente de plano.

Estas vicisitudes exigen que el problema sea abordado cartográficamente. Sobre todo por la necesidad de verificar la correspondencia entre la ciudad existente y los planes descritos por el intendente y sus colaboradores. En tal sentido, un documento fundamental, y que hasta ahora no se había utilizado, es el informe que Vicuña Mackenna encargó a Marcial Plaza (Plaza, I872), que definió los límites de la ciudad, basándose para esto en las subdelegaciones urbanas, a partir de las cuales describe sus límites y nombra cada una de sus calles ${ }^{4}$ (FIG. 3 y 4). En este sentido, el informe de Marcial Plaza permite hacerse una idea, relativamente precisa, de la extensión de la ciudad o de, al menos, lo que se entendía por esta en términos administrativos.

Aunque en casi todos los informes evacuados por las distintas comisiones encargadas de los estudios de factibilidad ${ }^{5}$ se habla de planos que los acompañan, por alguna razón, estos documentos no han llegado hasta nosotros. Esta ausencia de registros gráficos realza sin embargo el valor de los informes como fuentes documentales de primera mano, ya que gracias a sus descripciones es posible traducirlos a dibujos. Este es un punto sumamente importante, ya que reposiciona el dibujo como forma de conocimiento, cuestión que en aquel entonces ya estaba bastante clara, partiendo por
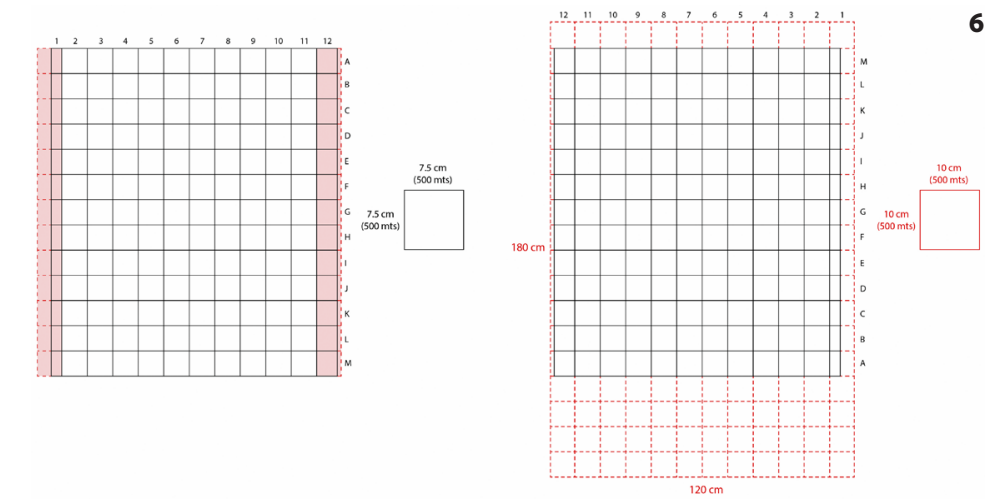

el mismo Vicuña Mackenna, quien siempre demostró un gran interés por los planos históricos de Santiago, aunque cuestionaba su valor técnico. Aun así, su insistencia en nombrarlos y organizar exposiciones para exhibirlos $^{6}$ es una clara prueba de lo que decimos. En fin, lo importante es que todo apuntaba a la innegable necesidad de un "plano definitivo" sobre el cual planificar la transformación.

Para decirlo en pocas palabras, que la ciudad fuera dibujable era para Vicuña Mackenna una señal de garantía de la posibilidad de materializar la transformación. Otra, y totalmente ligada a lo anterior, era la necesidad de que la ciudad tuviera una planta urbana clara y reconocible. En efecto, cuando en sus escritos Vicuña Mackenna se refería a la planta de la ciudad (I857) aludía a su forma, es decir, aquella dimensión urbana que sólo se puede visibilizar con precisión a través de un dibujo, en este caso, en una cartografía urbana que permite cotejar, simultáneamente, el plano y el plan.

\section{Plano-plan: un desafío cartográfico de ayer y de hoy}

Para realizar la nueva cartografía que presentamos con este artículo se han considerado los hechos básicos de la morfología de la ciudad en torno a I872: la trama de calles y el parcelario. Para ello se utilizaron fuentes primarias empíricas y detalladas de época posterior, como los planos de calles de Alejandro Bertrand de $1890^{7}$, el catastro de manzanas de $19 \mathrm{IO}^{8}$ y el levantamiento del valle de Santiago del Instituto Geográfico Militar de I9IO-I5․ La actualización a I872 se realizó, fundamentalmente, en base a la transcripción del informe realizado por Marcial Plaza (1872). El método para elaborar esta cartografía se funda, así, en la llamada ley de permanencia del plano, en que coinciden Pierre Lavedan (I926) y Marcel Poëte (20II). De este modo, la trama y los anchos de calles quedaron empíricamente validados. La incorporación de la subdivisión predial es sin lugar a dudas uno de sus elementos centrales, ya que evidencia con claridad el proceso de expansión que en ese entonces experimentaba la ciudad, a partir del cual se abrieron y crearon nuevas calles.

Todo lo anterior ha implicado un importante desafío cartográfico similar, en parte, al que afrontaron Vicuña Mackenna y Ernesto Ansart cuando propusieron un "plano definitivo" de Santiago. En este contexto, el plano actual se definió recogiendo e integrando algunas de las
FIGURA 5

Diagrama comparativo entre el "Plano

Definitivo" de Santiago de Benjamín Vicuña Mackenna y Ernesto Ansart, a escala 1:1.000 (izquierda), su reducción a escala 1:5.000 (centro), y el plano de Santiago de Ernesto Ansart, de 1875 (derecha).

\section{FIGURA 6}

Cuadrícula y coordenadas del plano de Santiago de 1875 de Ernesto Ansart y del plano definitivo, reducido, escala 1:5.000. Fuente: elaboración propia. Fondecyt N 1191393.

disminución del área urbana en relación a 1872. Además, el Camino de Cintura circunscribió la ciudad capital y la diferenció de los suburbios. 5. Vicuña Mackenna nombró diversos asesores y colaboradores. Para el Camino de Cintura, constituyó cuatro comisiones, una por cada sección. En la sección norte trabajó Ricardo Marín; en la poniente, Alfredo Cruz, Ramón Picarte y Guillermo Mackenna; en la sur, Joaquín Cortés, Domingo Toro Herrera, José Olano y Javier Ovalle y Vicuña; y en la oriente, José Manuel Figueroa y Eduardo

de la Barra. Se constituyó una ción norponiente, a cargo de Sinforiano Ossa. En todos esy en el informe de la Comisión General se menciona un plano general, además de planos por secciones. En la canalización del río, Ansart trabajó con el empresario Luis Cousiño, generando uno de los pocos dibujos que se conservan. Tras la muerte de Cousiño, Ansart colaboró con J. F. Gana, P. N. Marcoleta, S. Ossa y B. Ossa, y el mismo Vicuña Mackenna El estudio de la Alameda lo asumió Ansart y Marcial Plaza, mientras que en el Paseo del Santa Lucía colaboró con el arquitecto M. Aldunate. Por último, Ansart elaboró propuestas para la Avenida del Cementerio y Marcial Plaza realizó estudios para la apertura y prolongación de calles. comisión especial para la sectos casos se mencionan planos 


\section{CANALIZACION DEL MAPOCHO}

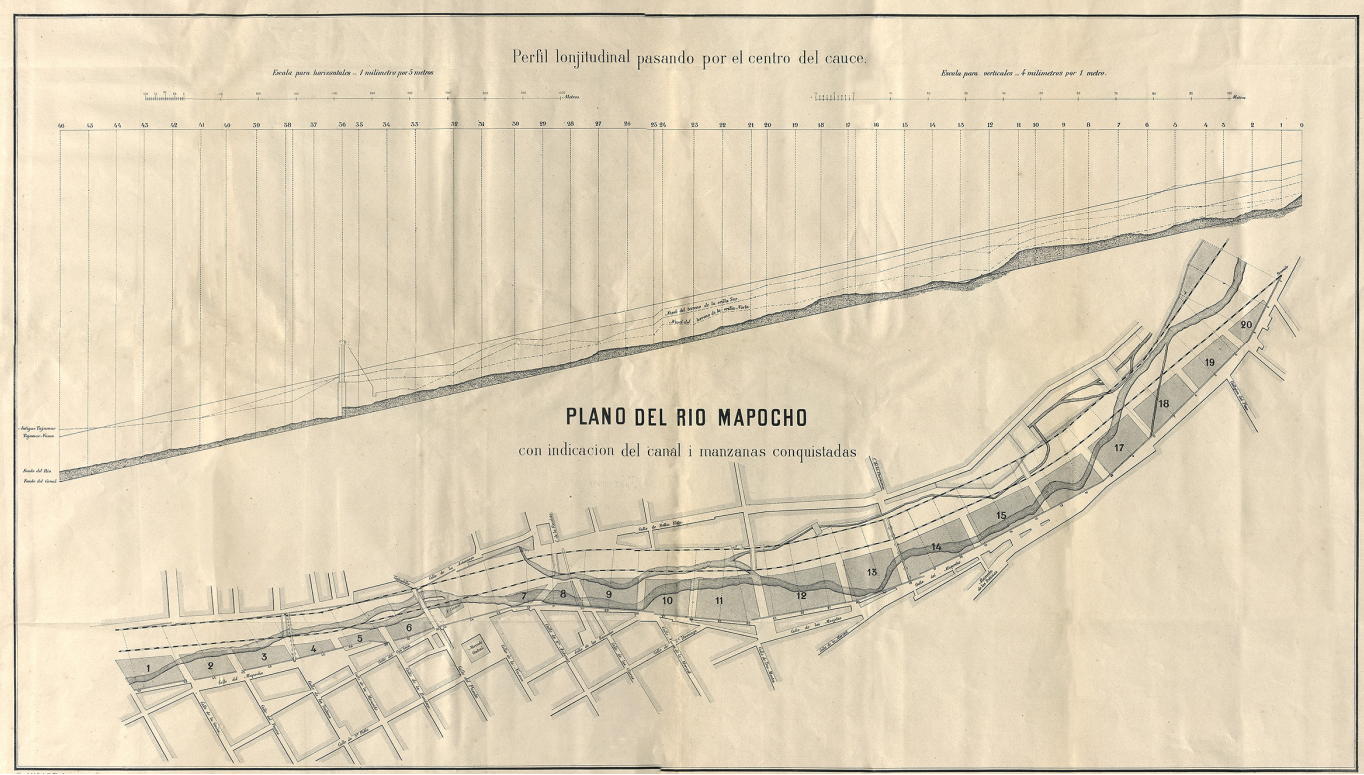

cualidades de ese plano que no fue. En parte también para visualizar sus rasgos más característicos.

Así, se ha mantenido la escala, i: 5.000, que corresponde a la que habría tenido el "plano definitivo" reducido (FIG. 5). Ello viene a confirmar que la escala I: 5.000 es la más apropiada para visualizar los principales elementos urbanos: la edificación, la subdivisión predial, los espacios públicos y el paisaje o ruralidad. El formato, por su parte, es una consecuencia directa de lo anterior, siendo su área útil de I, 8 × I,2 m; misma medida del plano definitivo reducido. La cuadrícula, que divide el territorio en áreas de $500 \times 500 \mathrm{~m}$, fue tomada directamente del plano de Ansart de I875, en el supuesto de que era la que estaba contemplada para el plano definitivo, toda vez que el ingeniero la incorporó en su plano, a pesar de que no calzaba con el formato ${ }^{\text {Io }}$ (FIG. 6). Finalmente, el encuadre fue ajustado a las proporciones resultantes de las medidas adoptadas: 3/2. De este modo, el alto del plano se distribuyó equitativamente en base a los tres cursos de agua fundamentales (el río Mapocho, el canal de San Miguel y el Zanjón de la Aguada) y sus suelos anexos. En consecuencia, el límite norte quedó definido por el Cementerio General y su entorno, mientras que el límite sur quedó definido por el Llano de Subercaseaux: un paseo que entonces ya era parte de la ciudad y cuyos terrenos habrían sido aportados por la familia de Victoria Subercaseaux, esposa de Vicuña Mackenna. En el ancho, de dimensiones más ajustadas, se dio cabida a las secciones oriente y poniente del Camino de Cintura, tal como, presumiblemente, lo consideraron Vicuña Mackenna y Ansart.

La parcelación urbana y rural fue representada con especial cuidado, ya que es el principal problema urbano de la época y por tanto es un tema clave de esta nueva cartografía. Como ya se dijo, para su definición se utilizó el catastro de I9Io y el levantamiento del Instituto Geográfico Militar (IGM), fuentes que se cotejaron con el plano de Santiago de $1850^{\text {II }}$ en un método retrospectivo. Para la identificación de los nuevos predios se hizo un estudio detallado del Catálogo de Bienes Raíces del Archivo

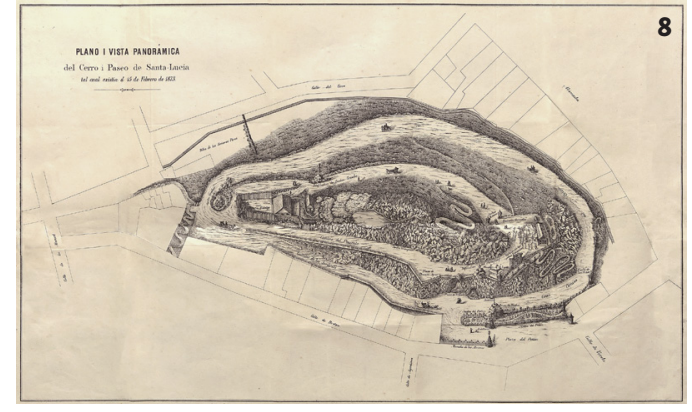

Nacional. Esta documentación, compuesta de inscripciones históricas de propiedad, permitió una aproximación a la situación en que se encontraba la ciudad hacia I872 y los años inmediatos ${ }^{12}$.

El plano se completó con aquellos elementos que logran dar cualidad tanto a la ciudad existente como a la propuesta. Se incorporó la topografía general junto a los canales y acequias principales. Las calles fueron representadas con su red de tranvías. Por ser un elemento primordial para caracterizar las nuevas avenidas, se incorporó la arborización, representada a través de puntos. La arquitectura, aunque no fue un elemento central en el plan de transformación, permite caracterizar los nuevos espacios públicos. Por esta razón, se consideraron los casos consignados por Ansart en su plano de I875, ya sea a través de la planta, de una imagen o de su pura denominación. Sin embargo, en el nuevo plano, todas estas representaciones se homologaron, dibujándose sólo plantas, con el mismo esquematismo que empleó Ansart. En aquellos casos en que Ansart no dibujó la planta del edificio se acudió a diversos planos históricos, particularmente el de Mostardi-Fioretti de $1864^{13}$, de donde el mismo Ansart los copió, en algunos casos.

Los proyectos representados en el plano fueron la canalización del Mapocho, de acuerdo a la versión dibujada por Ansart en I873 y que elaboró con Luis Cousiño, quien financió el estudio de factibilidad ${ }^{\text {I4 }}$ (FIG. 7); el Paseo del Santa Lucía que fue dibujado siguiendo distintas fuentes, particularmente, el Álbum del Santa Lucía de $\mathrm{cm})$. Fuente: Mapoteca de la Biblioteca Nacional de Chile.

\section{FIGURA 8}

"Plano y Vista panorámica del cerro Santa Lucía, tal cual existía el 15 de febrero de 1873 " $(49,5 \times 32,5 \mathrm{~cm})$ Fuente: Mapoteca de la Biblioteca Nacional de Chile.

6. Exposición Nacionalde Arte e Industria de I872, Santiago de Chile: Imprenta de la República, I873.

7. Fondecyt $\mathrm{N}^{\circ}$ iIIo684 "Santiago 1890. La calle como soporte y tránsito hacia la modernidad. Transcripción y montaje planimétrico del catastro de calles de Alejandro Bertrand" (2OII-2OI4).

8. Fondecyt $\mathrm{N}^{\circ}$ Io85253. "Santiago I9Io. Construcción planimétrica de la ciudad pre-moderna. Transcripciones entre el fenómeno de la ciudad física dada y la ciudad representada" (2008-20II).

9. Instituto Geográfico Militar, Santiago (Santiago, Chile: Instituto Geográfico Militar, I9IO-I9I5).

Io.El plano de Santiago de Ernesto Ansart mide $88,5 \times$ IOI, $5 \mathrm{~cm}$, y su escala es de I: 6.666 .

II. Fondecyt N ${ }^{\circ}$ II51318, "Santiago I850: la capital antes de su modernización. La mirada urbana de la expedición naval astronómica norteamericana de J. M.

Gilliss" (2015-2018).

I2. Los nombres de los propietarios, identificados en diferentes textos, como el estudio de Armando de Ramón, permitieron trabajar en el archivo del Conservador de Bienes Raíces y definir el régimen de propiedades para I872. El plano de igio, por su parte, permitió ubicar las propiedades aludidas.

I3. Teófilo Mostardi-Fioretti, Plano topográfico de la ciudad de Santiago de Chile (Santiago, I864).

I4. Ernesto Ansart,

Canalización del Mapocho perfil lonjitudinal pasando por el centro del cauce

(Vicuña Mackenna, 1874a). El plano iba acompañado de los perfiles del río y detalles del nuevo tajamar. Estos son prácticamente los únicos dibujos que han llegado hasta nosotros. 
I874 (Vicuña Mackenna, I874b) y el plano que contiene una "vista panorámica" del proyecto, atribuido a Ansart y Manuel Aldunate (Vicuña Mackenna, I873b) ${ }^{15}$ (FIG. 8). El Camino de Cintura fue, sin lugar a duda, el proyecto más cuidadosamente estudiado y transcrito dada su alta complejidad. Para ello se realizaron estudios especulativos, intentando interpretar a través de dibujos sus distintas versiones (Vicuña Mackenna, I872); de esa misma fuente se extrajo la información sobre la apertura de calles. En el caso del Camino de Cintura se optó por incluir todas las alternativas sugeridas por las comisiones. Las plazas, consideradas en los encuentros de las respectivas secciones, no fueron dibujadas por falta de antecedentes, con excepción de aquella de la sección poniente (segunda alternativa). En relación a la remodelación de la Alameda, esta se documentó desde Un año en la intendencia de Santiago (Vicuña Mackenna, I873a), siendo el trazado propuesto una transcripción de un texto escrito por Ansart ${ }^{16}$. Por último, también se incorporó en el plano el proyecto de la Avenida del Cementerio, propuesto también por Ansart, el cual consideró cinco alternativas ${ }^{17}$ (FIG. 9).

En síntesis, la cartografía que presentamos representa el posible estado de la ciudad Santiago hacia I872 y, sobre ella, las principales propuestas que Vicuña Mackenna ideó junto a sus colaboradores entre I872 y I875. Con ello se ha querido discutir tanto el problema teórico implícito en este tipo de cartografía, que aúna plano y plan en un mismo soporte de representación, como la problemática intrínseca que supone simultanear idea y realidad.

\section{Consideraciones finales}

Este trabajo se ha realizado con el objeto de develar la ciudad que el plano de Santiago de Ernesto Ansart, de I875, oculta o no permite ver, y lograr con ello una mejor comprensión de un período fundamental de la historia urbana de Santiago, identificado tradicionalmente con la intendencia de Vicuña Mackenna, que sin lugar a dudas marcó un punto de inflexión en la forma general de la ciudad.

A partir de esto y basándonos en la ambición de entonces, de realizar el "plano definitivo" de Santiago, nos hemos propuesto reconstruirlo para efectuar una lectura crítica y evaluar sus consecuencias en la ciudad actual. Tal como lo revela el plano que acompaña a este artículo, y aun cuando muchos de los cambios se implementaron años después, resulta innegable la influencia que tuvieron algunas de estas operaciones en la definición de su futura forma general. En específico, se debe considerar la propuesta del Camino de Cintura, ya que contenía el principio de lo que serían las grandes avenidas del siglo $\mathrm{xx}$.

Aun cuando quedó como una operación inconclusa -probablemente como consecuencia de no haber contado con el plano definitivo-, la realización de algunas secciones del Camino de Cintura, al modo de tramos lineales, constituyó una inequívoca pauta de ordenamiento futuro de la ciudad. Es preciso insistir en el carácter autónomo en que derivó cada una de sus secciones, pues, a la larga, fueron la pauta de un

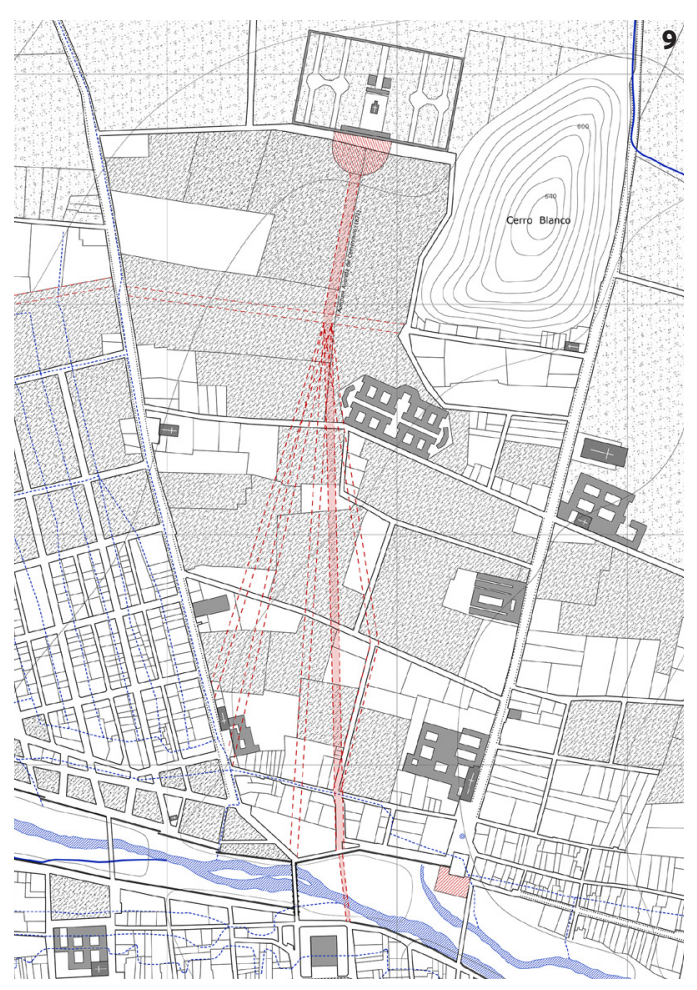

FIGURA 9

Propuestas para el trazado de la Avenida del Cementerio. Fuente: elaboración propia. Fondecyt $\mathrm{N}^{\circ}$ 1191393 a partir del estudio de Amilcar Garrido, estudiante de arquitectura UC. trazado que efectivamente rodeó la ciudad de forma continua, con el ferrocarril de circunvalación que se concretó a fines de siglo. Es posible afirmar que la complejidad de las zonas norte y poniente de la ciudad, que exigían un conocimiento detallado, fue la causa de esta incompletitud. Es más, ello es explicativo de la diversidad de alternativas generadas y de la indecisión final para concretar estas secciones.

Y es que el "plano definitivo" debía ser un instrumento de mediación entre la realidad existente y las verdaderas posibilidades de ejecutar las obras necesarias. Como ya se dijo, la mayor ambición de Vicuña Mackenna era dar a Santiago una nueva planta urbana y con ello lograr esa mediación, ese acuerdo tan fundamental como necesario en lo social y en lo urbano. En este sentido, el plano definitivo representaba la voluntad de ejecutar y documentar este pacto. De hecho, la versión oficial del plano, es decir, la representada a escala i: I.ooo, debía ser guardada y preservada para registrar las modificaciones futuras de la ciudad.

El dibujo del plano de Santiago, en la escala de baja proporcionalidad, al que se aspiraba exigía un levantamiento acucioso que diseccionara la organización espacial en su desarrollo urbanístico y arquitectónico, así como los distintos procesos que se encontraban en curso. Lo que aquí se ha evidenciado, es el nivel de evolución histórica registrado en un período muy concreto de la ciudad y aquellas operaciones que permitieron su modificación sustancial. Cuestión sólo constatable a través del nuevo plano realizado. Por último, representar en un plano-plan la forma general de la ciudad, al mismo tiempo que reforzar la organización del territorio que mediara entre la realidad urbana conformada por partes o fragmentos y la abstracción, finalmente terminó por ofrecer una nueva imagen para Santiago. De allí su importancia y trascendencia en la lectura de la ciudad actual.
I5. Además de la planta del proyecto se publicó un levantamiento topográfico del cerro realizado por Elías Marquez de la Plata en I869, lo que revela un interés anterior en desarrollar el cerro como paseo.

I6. El estudio de la Alameda fue realizado por lan Saintard, alumno del taller de investigación "Ernesto Ansart: el plano y el proyecto Transformaciones en Santiago de Chile, 1872-1875". Santiago: Escuela de Arquitectura, Pontifici Universidad Católica de Chile, segundo semestre de 2019.

I7. El estudio de la avenida del Cementerio fue realizado por Amilcar Garrido, alumno del taller de investigación "Ernesto Ansart: el plano y el proyecto Transformaciones en Santiago de Chile, I872-I875". 


\section{Bibliografía}

astaburuaga, Ricardo. "La ciudad de Vicuña

Mackenna”. En La montaña mágica. El cerro Santa Lucía y la ciudad de Santiago. Santiago: Ediciones ARQ, I993.

BARros arana, Diego. "Revista bibliográfica”. Revista Chilena (1875). Biblioteca Nacional Digital.

Cicuttı, Bibiana (ed.) La cartografía como objeto de cultura. Materiales para una discusión. Buenos Aires: Ediciones A\&P, 2012.

CORREA, Magdalena. "Proyectos para la transformación urbana de Santiago: la búsqueda de un nuevo orden y la consolidación del urbanismo como una disciplina profesional (I872-I934)”. Tesis para optar al grado de Magíster en Historia, Pontificia Universidad Católica de Chile, 2015.

DE RAMÓN, Armando. "Estudio de una periferia urbana: Santiago de Chile I850-I90o". Historia 20 (I985): 199-289.

FABELUKe, Graciela. El plano de la ciudad: formas y culturas técnicas en la modernización temprana de Buenos Aires (I750-I870). Buenos Aires: Instituto de Arte Americano, UBA, 202I.

HARDOY, Jorge. "La cartografía urbana en América Latina durante el periodo colonial. Un análisis de fuentes". En HARDoy, Jorge (ed.). Ensayos histórico-sociales sobre la urbanización en América Latina. Buenos Aires: Ediciones SIAP, I978.

HIDALGO, Germán; STRABUCCHI, Wren; MONTALbÁN, Magdalena. "Santiago I875: Una nueva edilidad. Los planes de la Intendencia de Vicuña Mackenna y el plano de Ernesto Ansart”. Revista I80 46 (2020): 6I-74.

LaVEDan, Pierre. Qu'est-ce que l'Urbanisme? Introduction à l'histoire de l'Urbanisme. París: Henri Laurens editeur, I926.

LEÓn echalz, René. Historia de Santiago. Santiago: Municipalidad de Santiago, I975.

MONTEAlegre, Pía. "La figuración del jardín público. urbanismo y agricultura en la construcción del Santiago moderno (I838-75)". Tesis para optar al grado de Doctora en Arquitectura y Estudios Urbanos, Pontificia Universidad Católica de Chile, 2015.

parcerisa, Josep. Forma Urbis. Cinco ciudades bajo sospecha. Barcelona: Laboratorio de Urbanismo, 2012.

parCERISA, Josep; SOLÁ-MORALEs, Manuel. La identidad del territorio. Barcelona: Quaderns d'Arquitectura i Urbanisme, I980.

PLAZA, Marcial. Santiago administrativo. Santiago: Editorial sin identificar, I872.

POËTE, Marcel. Introducción al Urbanismo La evolución de las ciudades. La lección de la antigüedad. Barcelona: Fundación Caja de Arquitectos, 2011.

RODRíguez, Hernán. "El intendente Vicuña Mackenna. Génesis y proyección de su labora edilicia”. Boletín de la Academia Chilena de la Historia 95 (I984): I03-I6o.

ROdríguez, Hernán. "La transformación de Santiago. Vicuña Mackenna I875”. Revista CA 8I (I995): 26-3I. vicuña maCkenna, Benjamín. "La ciudad de Santiago. I. Su presente, Il. Su pasado, Ill Su futuro." El mensajero de la Agricultura. Boletín de La Sociedad Nacional de Agricultura, 1857.

viCuÑa MACKenna, Benjamín. La transformación de Santiago. Santiago: Imprenta de la librería del Mercurio de Tornero y Garfias, I872.

vicuña MACKEnNa, Benjamín. Un año de la Intendencia de Santiago. Lo que es la capital y lo que debería ser. Vols. I y 2. Santiago: Imprenta de la librería del Mercurio de Tornero y Garfias, I873 (a).

vicuña mackenna, Benjamín. El Paseo de Santa Lucía, lo que es i lo que deberá ser. Santiago: Imprenta de la librería del Mercurio de Tornero y Garfias, I873 (b).

VICUÑA MACKENNA, Benjamín. La verdadera situación de la ciudad de Santiago. Santiago: Imprenta de la Librería del Mercurio, I874 (a).

vicuña mackenna, Benjamín. Álbum del Santa Lucía : colección de las principales vistas monumentos, jardines, estatuas i obras de arte de este paseo: dedicado a la Municipalidad de Santiago por su actual presidente B. Vicuña Mackenna. Santiago: Imprenta de la Librería del Mercurio, I874 (b).

vyHMEISTER-FÁbregas, Katherine. "La transformación de Santiago: un caso frustrado de intervención urbana a gran escala (I872-I929)”. EURE 45, no I45 (2019).

\section{Germán Hidalgo}

Arquitecto, Pontificia Universidad Católica de Chile, 1991. Doctor en Teoría e Historia de la Arquitectura ETSAB, 2000. Profesor Titular FADEU UC.

\section{Wren Strabucchi}

Arquitecto, Pontificia Universidad Católica de Chile, 1985. Ph.D en Filosofía y Arquitectura, Universidad de Cambridge, Inglaterra, 2001. Profesor de talleres y cursos de postgrado de arquitectura y paisaje. Investigador en cartografía urbana de Santiago.

\section{José Rosas}

Arquitecto y magíster Planificación Urbano Regional, Pontificia Universidad Católica de Chile. Doctor en Arquitectura ETSAB. Profesor Titular FADEU UC.

\section{Magdalena Montalbán}

Historiadora, Pontificia Universidad Católica de Chile. Magíster en Historia de la Arquitectura en la University College London. Estudiante de Doctorado uc. Ayudante de investigación del proyecto Fondecyt $n^{\circ} 1191393$.

\section{Germán Guzmán}

Arquitecto y máster en Arquitectura del Paisaje, Pontificia Universidad Católica de Chile. Profesor de pregrado de la Escuela de Arquitectura en el área de Construcción, Taller y Representación. 